\title{
Adaptive Dijkstra's Search Algorithm for MIMO detection
}

Original Scientific Papers

\author{
Karima Boukari \\ Badji-Mokhtar University \\ Faculty of Engineering, Department of electronics, \\ Laboratory for Study and Research in Instrumentation \\ and Communication Annaba (LERICA), \\ Annaba University P.B. 12, 23000, Annaba, Algeria. \\ boukkarima@yahoo.fr
}

\begin{abstract}
Employing Maximum Likelihood (ML) algorithm for signal detection in a large-scale Multiple-Input-Multiple-Output (MIMO) system with high modulation order is a computationally expensive approach. In this paper an adaptive best first search detection algorithm is proposed. The proposed Adaptive Dijkstra's Search (ADS) algorithm exploits the resources available in the search procedure to reduce the required number of nodes to be visited in the tree. A tunable parameter is used to control the number of the best possible candidate nodes required. Unlike the conventional DS, the ADS algorithm results in signal detection with low computation complexity and quasi-optimal performance for systems under low and medium SNR regimes. Simulation results demonstrate a $25 \%$ computational complexity reduction, compared to the conventional DS.
\end{abstract}

Keywords: Adaptive Dijkstra's algorithm, Maximum likelihood (ML) decoding, multiple-input, multiple-output (MIMO) systems, treesearch detection, optimization.

\section{INTRODUCTION}

In multiple-input multiple-output (MIMO) communication systems, each the transmitters and receivers are equipped with many antennas which can facilitate in achieving high gains in spectral, power, and energy potency compared to standard single-input singleoutput (SISO) systems wherever both the transmitters and receivers have just one antenna each. As a matter of fact, the MIMO systems have the power to show multipath propagation and multipath delay unfold into a profit for the receiver. Multiple Input Multiple Output (MIMO) system [1] is one of the major technologies adopted by current wireless communication standards such as Third Generation Partnership Project LongTerm Evolution (3GPP LTE), IEEE 802.11n, IEEE 802.16e as well as IEEE 802.11ac. Moreover, MIMO techniques will be used for the next generation of wireless technology systems (i.e., 5G), by increasing the number of antennas at the base station end. Employing the MIMO technology results in an increa-sed data rate and reliability of the communication systems [2], without compromising the bandwidth or the signal power. The key advantage of MIMO systems is the many orders of magnitude of the signal-to-noise ratio (SNR) at no additional bandwidth. However, it results in additional signal processing and computations in the receiver. At the receiver side, coming up with reliable and energyefficient MIMO detectors is a difficult task, as a result of the complexness of the implementation of the reception due to the interfering sub-streams. The signal detection problem refers to finding the most probable transmitted symbols based on the perfect channel state information available at the receiver and the received signal. Hence, the application of MIMO technology in real-time systems is expanded and has to be constrained with such additional computational complexity, while providing an intended quality of service (QoS) [3]. To attain a possible enhancement in data rate, the detection problem of spatially multi-plexed MIMO signals has attracted many researchers' attention in recent years. The main aim of the reported research is to optimally recover the transmitted signals with a reasonable complexity for hardware imple-mentation. In response to that, a wide range of detec-tion techniques has been proposed in the existing literature, [4-6]. By an exhaustive search over all the possible combinations of the transmitted signals, the Maximum likelihood (ML) detection scheme, can be argued to be the best detection technique which may not be feasible for a higher dimension of MIMO system or high modulation order $[7,8]$.

To overcome the increasing computation complexity during detection, some techniques such as the linear detection algorithm family such as Zero-Forcing (ZF) Minimum Mean Squared Error (MMSE) [2] or the iterative detection using the Vertical Bell Laboratories 
Layered Space-Time (VBLAST) technique have been proposed. Using these techniques decreases dramatically the detection complexity compared to the ML technique, but at the expense of a significant decrease in the detection performance. Sphere detectors (SD) can be represented using a tree-like structure to examine candidates nearby to an initial first guess. This first guess is used as the center point of the sphere to examine points within a small radius of this point. Sphere detectors typically have complexity sub-exponentially proportional to the constellation set size and number of antennas [9]. Optimal massive detectors such as ML or SD are considered infeasible given their high computational complexity [10].

By representing the MIMO signal detection as a tree search [11], a wide range of optimal and suboptimal techniques have been proposed in the literature with different tradeoff results between performance and complexity. The tree representation reformulates the MIMO signal detection problem to find the shortest possible branch from the top to the bottom of the tree within the optimal detection schema. Some algorithms initially based on finding the shortest path [12], are now adapted for the MIMO detection problem. These techniques can be classified into three different categories of tree search: depth-first, breadth-first and best first. In the MIMO signal detection, based on a tree search, all the proposed algorithms aimed at reducing the number of visited nodes and the total computation cost required for establishing the optimal solution [13]. Dijkstra's algorithm $[14,15]$ reduces the number of visited nodes by maintaining a list of candidate nodes and equally search among them in the order of the best first. Despite the optimal performance obtained, the search involves visiting unnecessary nodes with equal importance to each node. Hence, the number of visited nodes and the memory requirement remain high for practical implementation of such an algorithm in systems with high modulation order.

In this paper, a new MIMO signal detection algorithm is proposed based on the best first search. The proposed algorithm is adaptive; it defines some criteria to reduce the number of the combinations to be evaluated and visits only the most likely combination to result in an optimal solution and hence, a large amount of calculation can be avoided. The proposed algorithm is an adaptive version of Dijkstra's algorithm with respect to transmit-receive antenna and modulation constellation dimension. The complexity reduction within the proposed algorithm is directly proportional to the modulation order with quasi-optimal performance in the low SNR regime.

The rest of the paper is organized as follows. Section 2 presents the system model and conventional Djikstra's algorithm. Then, the proposed algorithm is presented in section 3 . Simulation results and discussions are presented in section 4. Finally, some conclusions are given in section 5 .

\section{SYSTEM MODEL AND DIJKSTRA'S ALGORITHM}

\subsection{SYSTEM MODEL}

We consider a spatial multiplexed MIMO system with $\mathrm{N}_{\mathrm{t}}$ transmitter antennas and $\mathrm{N}_{\mathrm{r}}$ receiver antennas with Rayleigh fading channel, the received signal vector can be expressed as:

$$
\mathrm{y}_{\mathrm{c}}=\mathrm{H}_{\mathrm{c}} \times \mathrm{s}_{\mathrm{c}}+\mathrm{v}_{\mathrm{c}}
$$

where $y c$ is an $\left(\mathrm{N}_{\mathrm{r}} \times 1\right)$-dimensional vector representing the received signal and $\mathrm{sc}$ is an $\left(\mathrm{N}_{\mathrm{t}} \times 1\right)$-dimensional vector representing the transmitted signal. Its elements are drawn from a set of complex elements such as the $\mathrm{M}-\mathrm{QAM}$ constellation, where $\mathrm{M}$ is the modulation order, $\mathrm{H}_{\mathrm{c}}$ is an $\left(\mathrm{N}_{\mathrm{r}} \times \mathrm{N}_{\mathrm{t}}\right)$ matrix representing the channel with independent and are identically distributed (i.i.d.) Gaussian entries with zero mean and unitary variance and $\mathrm{vc}$ is $\left(\mathrm{N}_{\mathrm{r}} \times 1\right)$-dimensional vector of noise with an i.i.d. complex entries with zero mean and variance $\sigma_{n}^{2}$. For simplicity, the numbers of transmitting and receiving antennas are assumed to be symmetrical, i.e. $\mathrm{N}_{\mathrm{t}}=\mathrm{N}_{\mathrm{r}^{\prime}}$ the channel state information is assumed to be known to the receiver.

The equivalent real presentation of the system (1) is defined by

$\left[\begin{array}{c}\mathfrak{R}\left(\mathrm{y}_{\mathrm{c}}\right) \\ \mathfrak{I}\left(\mathrm{y}_{\mathrm{c}}\right)\end{array}\right]=\left[\begin{array}{cc}\mathfrak{R}\left(\mathrm{H}_{\mathrm{c}}\right) & -\mathfrak{I}\left(\mathrm{H}_{\mathrm{c}}\right) \\ \mathfrak{I}\left(\mathrm{H}_{\mathrm{c}}\right) & \mathfrak{R}\left(\mathrm{H}_{\mathrm{c}}\right)\end{array}\right] \times\left[\begin{array}{c}\mathfrak{R}\left(\mathrm{s}_{\mathrm{c}}\right) \\ \mathfrak{I}\left(\mathrm{s}_{\mathrm{c}}\right)\end{array}\right]+\left[\begin{array}{c}\mathfrak{R}\left(\mathrm{v}_{\mathrm{c}}\right) \\ \mathfrak{I}\left(\mathrm{v}_{\mathrm{c}}\right)\end{array}\right]$,

where $\mathfrak{R}(*)$ and $\mathfrak{I}(*)$ denote the real and imaginary parts of its elements. The equivalent real representation of the system model in (2) is presented as follows

$$
\mathrm{y}=\mathrm{H} \times \mathrm{s}+\mathrm{v},
$$

where $y \in R^{n}, s \in R^{m}, H \in R^{n \times m}$ and $v \in R^{n}$ with $n=2 \times N_{r}$ and $\mathrm{m}=2 \times \mathrm{N}_{\mathrm{t}}$

\subsection{MIMO DETECTION BASED ON TREE SEARCH}

In the MIMO detection, the maximum likelihood ML detection achieves the optimal bit error rate performance by solving the minimization problem

$$
\hat{\mathrm{s}}=\arg \min _{\mathrm{s} \in \Omega^{\mathrm{m}}}\|\mathrm{y}-\mathrm{Hs}\|^{2},
$$

where $\Omega$ is the constellation in the real-valued system model, for example, in $16-\mathrm{QAM} \Omega=\{+3,+1,-1,-3\}$.

The ML detection makes an exhaustive search over all the candidates of "s". Hence, the complexity of the detection increases exponentially with the number of antennas and the modulation order $\mathrm{M}$, making it impractical for real-time implementations. 
By applying the QR decomposition on the channel matrix, expression (4) is reformulated by the equivalent expression

$$
\hat{\mathrm{s}}=\arg \min _{\mathrm{s} \in \Omega^{\mathrm{m}}}\|\mathrm{y}-\mathrm{Hs}\|^{2}
$$

with $P(s)=\left\|y^{\prime}-R s\right\|^{2}$ and $y^{\prime}=Q^{H} R$, where $Q$ is a unitary matrix and $\mathrm{R}$ is an upper triangular matrix.

Because of the upper triangular matrix $\mathrm{R}$, the quantity $P(s)$ in (5) can be calculated in a recursive process as shown in (6). Thus, that is the MIMO detection is reformulated as follows:

$$
\mathrm{P}\left(\mathrm{s}_{k}^{l}\right)=\mathrm{P}\left(\mathrm{s}_{k-1}^{l}\right)+\mathrm{B}\left(\mathrm{s}_{k}^{l}\right)
$$

with $\mathrm{B}\left(\mathrm{s}_{k}^{\prime}\right)=\left(y_{k}^{\prime}-\sum_{k, j}^{m} r_{k, j} s_{j}^{2}\right.$, where $\mathrm{y}_{\mathrm{k}}^{\prime}$ and sj are the real elements of $y$ and ${ }^{j=k}$ respectively, and $r_{k, j}$ is the (k, j)-entry of $\mathrm{R}$.

The minimization problem represented in Eq. (5) is solved by a tree search with $\mathrm{m}$ layer(s) as depicted in Fig. 1. Each node in Fig. 1. represents a partial candidate symbol vector $\mathrm{s}_{\mathrm{k}}^{\mathrm{l}}$ which is weighted by two metrics; path metric $\mathrm{P}\left(\mathrm{s}_{\mathrm{k}}^{1}\right)$ and branch metric $\mathrm{B}\left(\mathrm{s}_{\mathrm{k}}^{1}\right)$. As illustrated in Fig. 1., the tree search procedure starts from layer $\mathrm{l}=0$, denoted with a dotted circle. It is named as a root node, which acts as a reference to calculate branch metric at layer $\mathrm{l}=1$. Solid circled nodes and shadedcircle represent the visited nodes and the leaf nodes respectively, where an estimation of the path metric is required to make a decision on a partial candidate vector or a candidate vector. In the tree search procedure, it is expected to visit the node and expand towards the child nodes if required.

For conventional ML signal detection with such a tree search algorithm, all the possible candidate nodes are required to be visited. A final decision on the detected symbol vector is the leaf node with the smallest path metric and is referred to herein as the ML solution. The objective of the optimization is to reduce the number of visited nodes and output the ML solution.

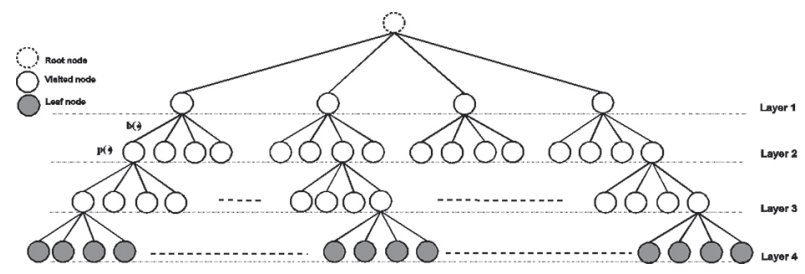

Fig. 1. Tree search for $2 \times 2$ MIMO system with 16QAM modulation $\mathrm{m}=2 \mathrm{xNt}$ and $\Omega=\{+3,+1,-1,-3\}$.

\subsection{DIJKSTRA'S TREE SEARCH ALGORITHM:}

Dijkstra's Search algorithm (DS) has been proposed initially for graph search [12]; later it has been applied to
MIMO signal detection [16-18]. As shown in Fig. 2., the conventional Dijkstra's algorithm visits all the nodes, at first layer $(l=1)$, by estimating their respective path metrics; then it selects the node with the smallest path metric to be the mother node, while the rest of the nodes are the siblings. All the possible nodes expanded from the mother node at $\mathrm{l}=2$ are considered as child nodes and their respective path metrics are calculated. In the next iteration of the search procedure, all the candidate nodes, consisting of the sibling's nodes and the new child nodes, are considered in the search of the new mother node. The new mother node is then expanded to its child nodes. This procedure continues until the selection of the mother node at the last layer $\mathrm{l}=\mathrm{m}$ which constitutes the detectable symbol of interest.

The DS algorithm was also proposed for systems with memory constraints [16]. In these systems, the size of the candidate nodes list is explicitly assigned and is equal to the maximum memory available to the system. The nodes with an index superior to the maximum list size are discarded. For the systems with memory constraint, a relevant of the Dijkstra's algorithm has been proposed in [19]. The algorithm limits the list of the candidate to choose from the mother node. and it is named in this paper $D S(U)$ with $U$ is the memory.

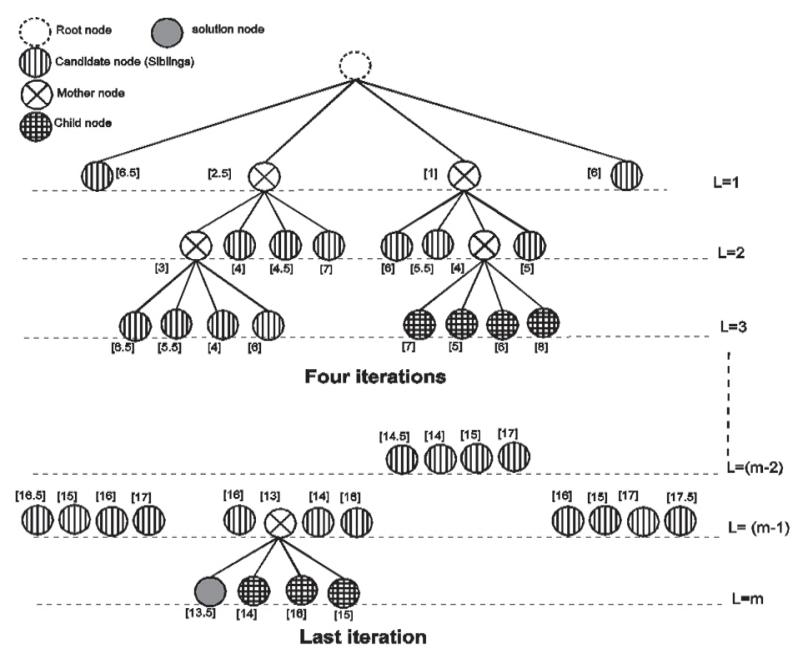

Fig. 2. Illustration of Dijkstra's search algorithm (DS) for MIMO signal detection based on tree search. The number between brackets represents the path metric $\mathrm{P}\left(s_{k}^{l}\right)$ of the node.

\section{Algorithm I: DS(L)}

A0. (Initialization) Given an initial node list $C$ that contains the root node only, and node list size constraint $L$.

A1. (Selecting the best node) Select the first node (the best node in this iteration) from $C$. If this node is in layer 1, stop the algorithm and output this node as the solution.

A2. (Expanding the best node) Expand the best node by adding all its children nodes to $C$ and removing itself from $C$. 
A3. (Maintaining and sorting the node list) Order the nodes in $\mathrm{C}$ in ascending order of their path metric. Retain the first $\min (|C|, L)$ nodes and discard others. Go to $A 1$.

\section{PROPOSED ADAPTIVE DIJKSTRA'S ALGORITHM (ADS)}

The computational complexity of the conventional DS algorithm is exponentially proportional to the number of nodes required to visit in the detection process. In this paper, we propose a novel candidate search that requires a lower number of nodes to be visited; subsequently, It's expected to that the computational complexity is reduced. The proposed algorithm is based on an adaptation criterion to select the optimum nodes to be visited and is referred to herein as Adaptive Dijkstra's Search algorithm (ADS).

For each iteration, the ADS performs three new steps:

\section{- STEP 1: Defining the number of child nodes}

The degree of closeness between the path metric of the mother node and the siblings is used as a selection criterion to define the number of child nodes to be visited and it is evaluated by exploiting two estimated parameters: $\gamma$ and $\mu$ calculated by

$$
\begin{aligned}
& \gamma=\frac{\mathrm{P}_{\mathrm{i}}(3)-\mathrm{P}_{\mathrm{i}}(1)}{2}, \\
& \mu=\mathrm{P}_{\mathrm{i}}(2)-\mathrm{P}_{\mathrm{i}}(1) .
\end{aligned}
$$

In the case of $\mu \geq \gamma$, the mother node is too small compared to the second smallest node in the candidate list (first sibling). It means that the current mother node and its child nodes are the most promising nodes while the siblings are less likely to lead to the ML solution. From this point of view, all the child nodes have to be visited. Otherwise $(\mu<\gamma)$, the mother node is comparable to the first sibling. In this case, each sibling is also expected to be viewed as a promising node to lead to the $M L$ solution. To enhance the possibility for the siblings to be expanded, the ADS is designed to reduce the number of child nodes that correspond to the selected mother.

The number of child nodes $w$ to be visited is defined by:

$$
w=\left\{\begin{array}{c}
|\Omega| \text { if } \mu \geq \gamma \\
0.25 \times c \times|\Omega| \text { if } \mu<\gamma
\end{array},\right.
$$

where $c=\{1,2,3\}$ is a tunable parameter used to predefine the number of child nodes to be visited in the ADS search procedure.

\section{- STEP 2: Ordering the child nodes}

If the number of child nodes required to be visited is less than $|\Omega|$, only the best child nodes will be concerned. A Symbol estimator is performed using the re- ceived signal vector, the channel state information, and the just detected signal to obtain an estimated symbol $\hat{s_{k}}$ expressed in (10). The output of the estimator for the respective mother node leads towards sorting the child nodes in the order of the nearest first as presented in Fig. 3.

$$
\hat{s_{k}}=\left\{\begin{array}{c}
y_{k} / r_{k, k} \\
\left(y_{k}-\sum_{i=k+1}^{m} r_{k, i} s_{i}\right) / r_{k, k} .
\end{array}\right.
$$

To avoid the additional computations of the sorting, a predefined look-up table (LUT), based on the output of the estimator, can be designed to select better/fitter child nodes.

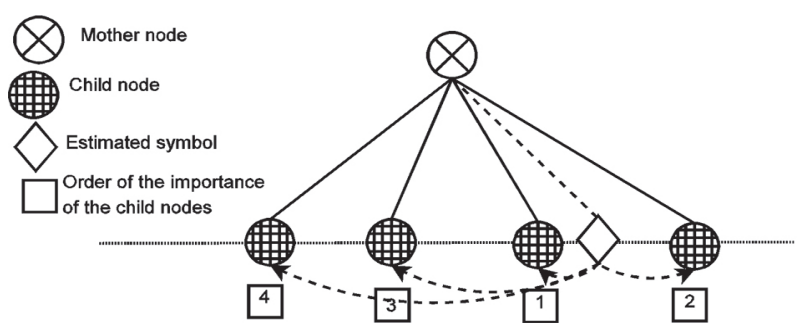

Fig. 3. Ordering the child nodes based on the estimated symbol exp: 16-QAM

\section{- $\quad$ STEP 3: Visiting the child nodes}

From the ordered child nodes, the proposed ADS algorithm visits only the $w$ best nodes.

Fig. 4. depicts the tree expansion of ADS algorithm. In the first iteration, the number of visited child nodes is reduced to two, while it is maintained to four in the second iteration. The search continues by defining the number of child nodes in each iteration until reaching the last layer.

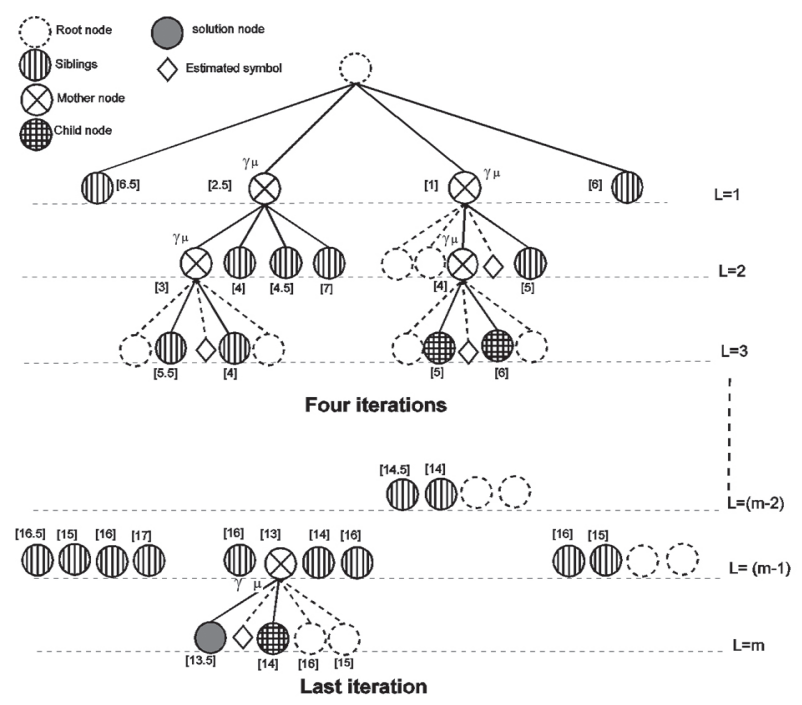

Fig. 4. Illustration of the Adaptive Dijkstra's search algorithm (ADS) for MIMO signal detection based on tree search. The number between brackets represents the path metric $\mathrm{P}\left(s_{k}^{l}\right)$ of the node. 
The proposed ADS is based on an adaptive search on each expanded node. It is more suitable for transmitted signals with high modulation order and with a given SNR, which result in an increased number of expanding nodes. Unlike the conventional DS, which visits all the child nodes, ADS visits adaptively a reduced number of child nodes. Subsequently, the ADS algorithm is expected to outperform the conventional DS algorithm in terms of computation complexity especially for systems with high modulation order.

The reduction of computational complexity due to the elimination of one child node is equal to the additional computational requirement due to the mother node expansion. In ADS algorithm, many more child nodes are more likely to be discarded by adjusting the proposed tunable parameter. Hence the reduction in computational complexity is expected to be significant compared to the conventional DS algorithm. These statements will be further demonstrated by our simulation results, presented in the following section.

\section{Algorithm II: ADS(L)}

A0-A1. Same as Algorithm I.

A2. (Defining the number of child nodes) calculate $\gamma$ and $\mu$ by (7) and (8). If $\mu \geq \gamma$, then all the child nodes have to be visited. Conversely, the number of child nodes $\mathrm{W}$ to be visited is defined by (9).

A3. (Ordering the child nodes) an estimated symbol sk expressed in (10) leads towards sorting the child nodes in the order of the nearest first.

A4. (Expanding the best node) Expand the best node by adding its $w$ children nodes to $C$ and removing itself from C.

A5. (Maintaining and sorting the node list) Order the nodes in $\mathrm{C}$ in ascending order of their path metric.

Retain the first $\min (|C|, L)$ nodes and discard others. Go to A1.

\section{SIMULATION RESULTS AND DISCUSSIONS}

\subsection{EXPERIMENT DESIGN}

This section aims to experimentally compare the efficiency of the proposed ADS algorithm over the conventional DS algorithm in MIMO signal detection, in terms of Symbol Error Rate (SER) performance (achieving the optimal solution for signal detection) and the associated computational complexity. MATLAB simulation environment is used to realize the simulations. Table I presents the different parameter settings for the experiments. The SER is employed to compare the performance of the systems with different signal detection schemes, while the average number of visited nodes and flops is calculated to evaluate the computational complexity.

In this experiment, a 4x4 MIMO system with 64-QAM modulation order is considered. Four detection algo- rithms were tested on this system, ZF, SD (Sphere Detection), DS and ADS. As for the memory, two scenarios were considered for both DS and ADS algorithms $(U=1$ and $\mathrm{U}=8$ ).

\subsection{RESULTS AND DISCUSSIONS}

In our study, we carried two experimentations, the first one, with $c=1$, where only $25 \%$ of nodes are visited and the second one with $c=3$, where $75 \%$ of nodes are visited.

Table 1. Simulated MIMO systems

\begin{tabular}{|cc|}
\hline Parameters & Values \\
\hline Number of transmit antennas & 4 \\
\hline Number of receive antennas & 4 \\
\hline Modulation order (M-QAM) & 64 \\
\hline Tunable Parameter (c) & $1(25 \%), 2(50 \%), 3(75 \%)$ \\
\hline Channel & Rayleigh \\
\hline
\end{tabular}

Figures 5 and 6 resume our results for $c=1$ and $c=3$ respectively. As shown in the two figures, the SD algorithm was employed to verify that the conventional DS has an optimal error performance.

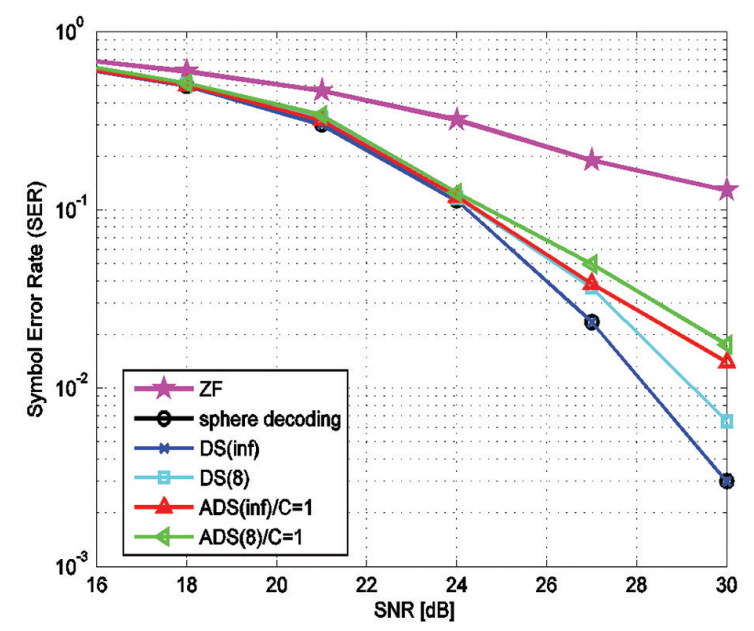

(a) SER performance

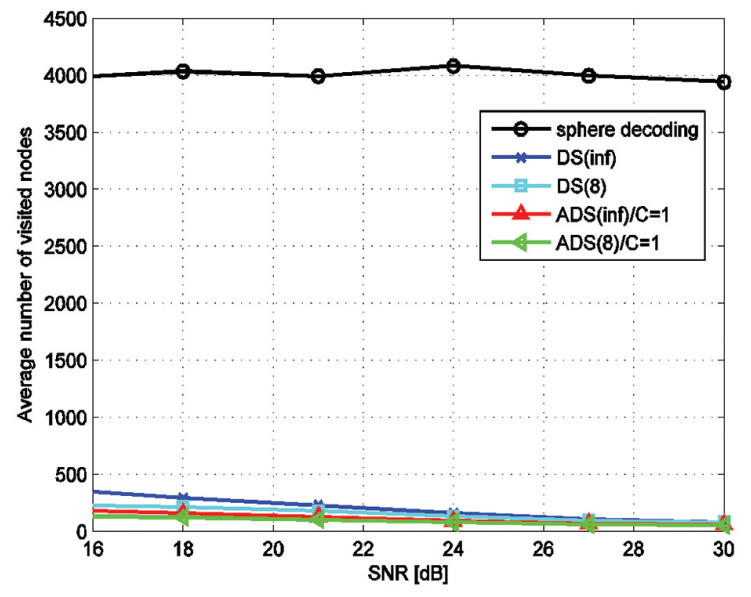

(b) Computation complexity 


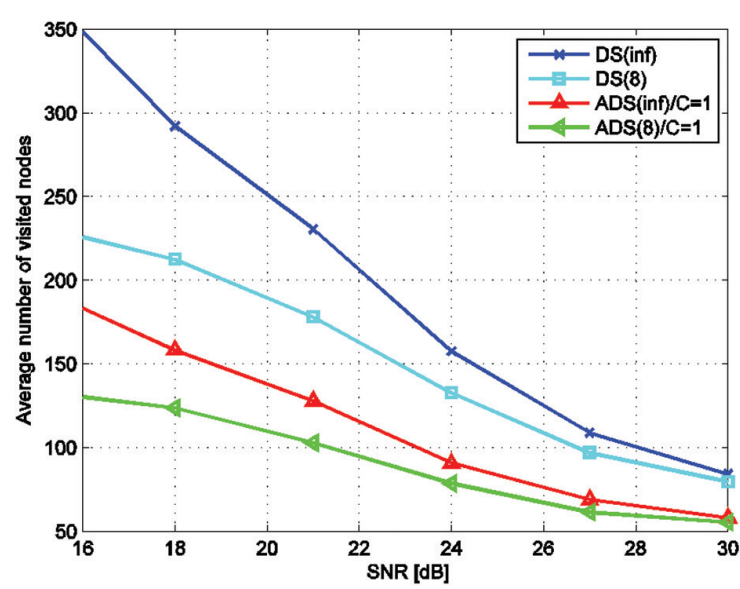

(c) Computation complexity (number of visited nodes)

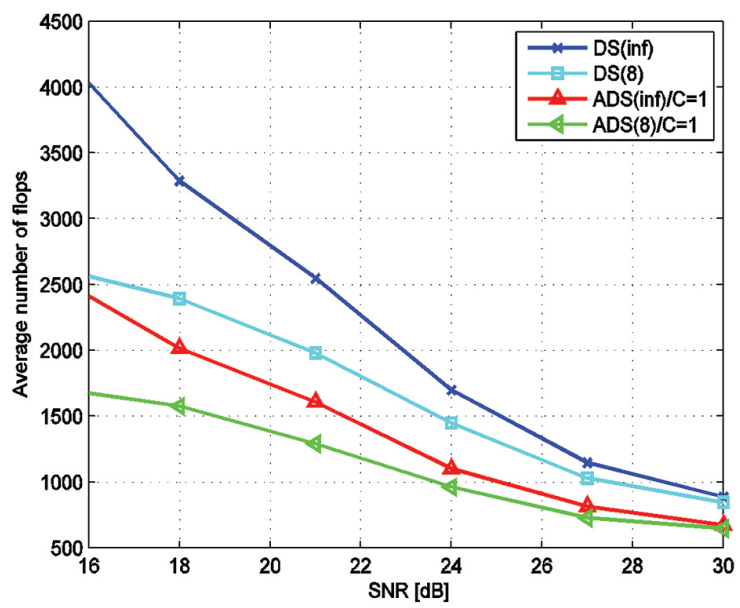

(d) Computation complexity (number of flops)

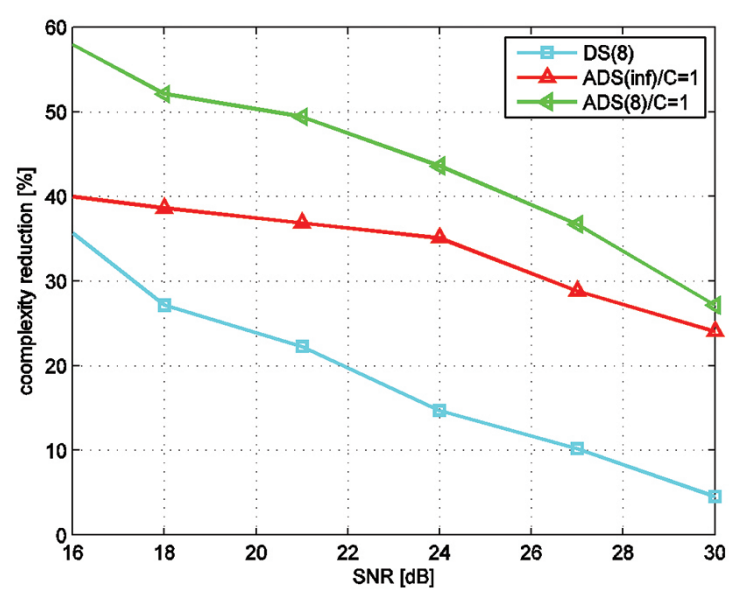

(e) Reduction of computation complexity

Fig. 5. Performance comparison of the ADS algorithm with $\mathrm{c}=1$ in $4 \times 4 \mathrm{MIMO}$ system and 64QAM modulation.

\section{- Experiment with $c=1$}

It is interesting to notice that in Fig. 5 -a for SNR $<24$ $\mathrm{dB}$, the performance of the conventional DS and the proposed ADS with constrained $(\mathrm{U}=8)$ and unconstrained memory $(U=1)$ is almost the same, with negligible differences. It is only when SNR $>24 \mathrm{~dB}$ that the performances of the different algorithms start to differentiate but gradually and only slightly following this sequence from best to worst: DS(1), followed by DS(8), then $\operatorname{ADS}(1)$, and lastly $\operatorname{ADS}(8)$. For $S E R=10^{-2}$, the performance of $A D S(1)$ is degraded by about $3 \mathrm{~dB}$ compared to the $D S(1)$ while the performance of $A D S(8)$ is degraded by about 2,5 dB compared to DS(8).

As presented in Fig. 5-b, the DS(1) reduces the number of visited nodes required to achieve the best solution by an average of 4200 nodes compared to sphere decoding. Fig. 5-c and Fig. 5-d present the number of visited nodes and flops, respectively, required to output the detected signal. The four graphs follow a similar trend with a large number of visited nodes and flops for low SNR and lower for high SNR. The proposed ADS(8) has the lowest number of flops, while ADS(1) requires fewer flops than DS(8) and ADS(8).

By referring to the computational complexity of the conventional DS(1), in Fig. 5-e, the proposed ADS(1) reduces the complexity by $25 \%$ to $40 \%$ While the ADS(8) reduces the computational complexity by $20 \%$ compared to $\mathrm{DS}(8)$.

The optimal curves of the SER and computational complexity are presented in contrast to the curves of the SER and the number of flops of the conventional DS(1) respectively.

For low and medium SNR:

1) The noise affects the transmitted symbols, which appear in the tree as nodes equally probable leading to the optimal solution and their path metrics are relatively close. Therefore, a large number of nodes have to be visited as presented in Fig. 5-c. Due to the equally searching of the DS(1) algorithm, all the child nodes are visited, even the weakest nodes, for example, 350 nodes are visited for $S N R=16 \mathrm{~dB}$.

2) On the contrary, the $A D S(1)$ visits the strongest child nodes ( $25 \%$ in this experiment) and discards a large number of the weakest child nodes (75\%). As a result, the total visited nodes is 180 for $\mathrm{SNR}=16 \mathrm{~dB}$.

3) Given a large number of the visited nodes, the probability associated with each node leading to the optimal solution has to be small, which results in a negligible SER degradation for discarding the weakest child nodes.

4) On the other hand, this pruning task reduces the computational cost (about 40\% compared to DS(1)) by conserving the calculations of the path metrics of the discarded child nodes.

For the memory constraint, the ADS inherits also the behavior of the conventional DS. The ADS(8) has the same SER performance as DS(8) while reducing the computations by $21 \%$. 
For high SNR:

1) The degraded performance of the $\operatorname{ADS}(1)$ compared to the DS(1) is caused by discarding the child nodes. Knowing that the mother node in this region of SNR is the most likely to end to the ML solution, reducing the number of its child nodes enhances the probability to discard the optimal solution. As a result, a high degradation of SER performance is observed where $75 \%$ of child nodes are discarded.

It is important to note that in cases when SNR is less

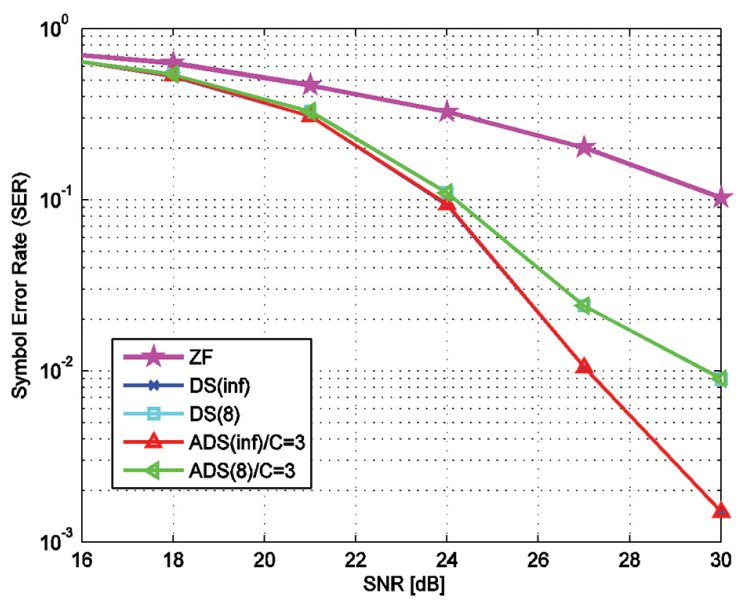

(a) SER performance

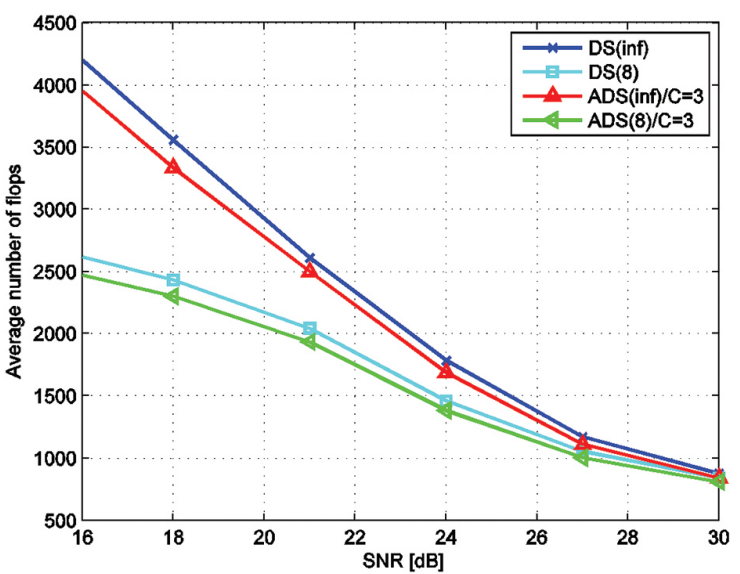

(c) Computation complexity (number of flops) than $24 \mathrm{~dB}$, visiting only $25 \%$ of child nodes, $A D S(1)$ with $c=1$, seems to be sufficient to outperform the DS(1) with quasi-optimal performance as well as a significant reduction of computation complexity by up to $40 \%$ observed.

\section{- Experiment with $\mathrm{c}=3$}

In Fig. 6, the SER performance and the computation complexity are calculated for the ADS algorithm with $c=3$ (75\% of child nodes are visited).

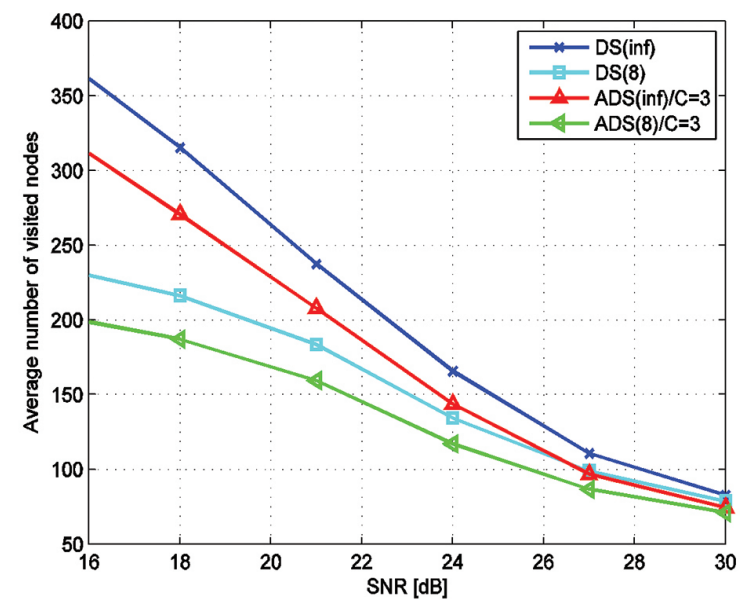

(b) Computation complexity (number of visited nodes)

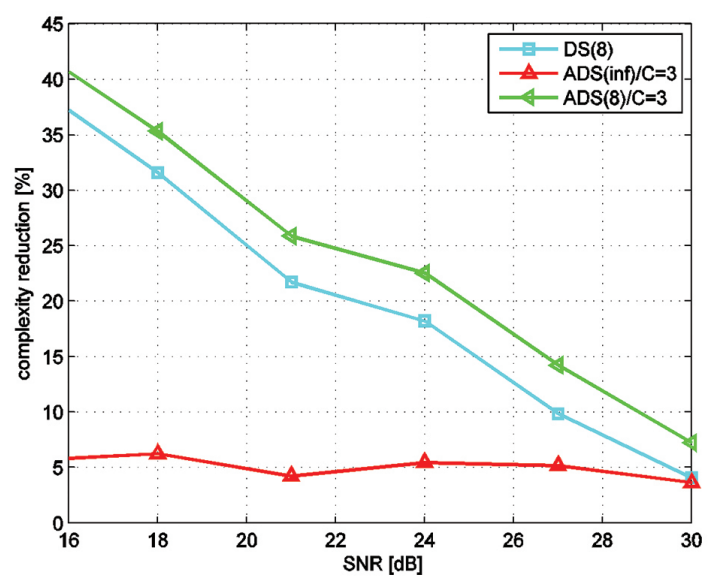

(d) Reduction of computation complexity

Fig. 6. Performance comparison of the ADS algorithm with $c=3$ in $4 \times 4$ MIMO system and 64-QAM modulation.

As presented in Fig. 6-a, both the performances of ADS(1) and ADS(8) are the same as the performances of DS(1) and DS(8), respectively, across all SNR levels. Fig. 6-b and Fig. 6-c present the number of visited nodes and flops, respectively, required to output the detected signal. The two graphs follow a similar trend with a large number of visited nodes and flops for low SNR and lower for high SNR. As an example, let's compare the number of nodes for $\mathrm{C}=1$ it goes from 350 (for DS(1)) to 180 (for ADS(1)), while for $c=3$ it changes from 360 (for DS(1)) to 320 (for ADS(1)). As expected, the spread is smaller for the number of nodes and for the flops. Hence the reduction in computational complexity is less significant. As presented in Fig. 6-d, The complexity reduction is about $5 \%$ for both ADS(1) and ADS(8) compared to DS(1) and DS(8), respectively. In summary, visiting $75 \%$ of the best child nodes conserves the optimality of the DS(1) algorithm and reduces its complexity by $5 \%$.

\section{CONCLUSION}

Due to the growing interest in large-scale MIMO systems for the future generation of wireless communication systems, the reduction of the computational complexity within ML detection schemes has been addressed in this paper. A tunable adaptation param- 
eter has been introduced while attaining the intended application-specific quality of service (QOS) as a tradeoff with computational complexity. More specifically, a novel tree search procedure has been proposed. The simulation results have been fairly consistent with the theoretical expectations and shows the potential superiority of the proposed ADS over the conventional DS. For real-world applications, the MIMO systems operate in low to medium SNR; where the proposed ADS proved to outperform the conventional DS. In this case, the results show that searching only $25 \%$ of the child nodes is sufficient to achieve an acceptable quasi-optimal solution, resulting in a computational complexity reduction of up to $40 \%$. For high SNR regions, such reduction in computational complexity is attained at a minimum cost of additional SNR. However, in the case of $75 \%$ of the tree search, the ADS outperforms the conventional DS across all SNR levels, yet again with some reduction in computational complexity. Adaptation of the dynamic modulation resolution as well as dynamic selectivity of active antennas within large scale MIMO system potential focus to attain an intended QoS consistently using the proposed detection scheme. This work focuses on the uncoded MIMO systems. It will also be interesting to adapt this work in the coded MIMO systems in the category of the soft MIMO detection algorithms. These algorithms are based on iterative detection by using the information of channel decoder. Such integration can be investigated to maximize the benefits of the MIMO systems.

In addition, even if the algorithms derived in this paper do not consider OFDM modulated techniques as an option. it may be included in future works. Finally, it's worth noting that any novel detection algorithm, that do not use the heuristic weight, is of great interest.

\section{REFERENCES:}

[1] A. Paulraj, D. Gore, R. Nabar, H. Bolcskei, "An overview of mimo communications - a key to gigabit wireless", Proceedings of the IEEE, Vol. 92, No. 2, 2004, pp. 198-218.

[2] F. Rusek, D. Persson, B. K. Lau, E. G. Larsson, T. L. Marzetta, challenges with very large arrays", IEEE Signal Processing Magazine, Vol. 30, No. 1, 2013, pp. 40-60.

[3] S. Yang, L. Hanzo, "Fifty years of MIMO detection: The road to large-scale MIMOs", IEEE Communications Surveys and Tutorials, Vol. 17, No. 4, 2015, pp. 1941-1988.

[4] F. Jiang, C. Li, Z. Gong, "Low Complexity and Fast Processing Algorithms for V2I Massive MIMO Uplink Detection," IEEE Transactions on Vehicular Technology, 2018, pp.1-15.
[5] J. Zeng, J. Lin, Z. Wang, "Low Complexity Message Passing Detection Algorithms for Large-Scale MIMO Systems", IEEE Wireless Communications Letters, 2018, pp. 1-4.

[6] S. Wu et al, "Low-Complexity Iterative Detection for Large-Scale Multiuser MIMO-OFDM Systems Using Approximate Message Passing", IEEE Journal of Selected Topics in Signal Processing, Vol. 8, No. 5, 2014, pp. 902-915.

[7] X. Zhu and R. Murch, "Performance analysis of maximum likelihood detection in a mimo antenna system", Communications, IEEE Transactions on, Vol. 50, No. 2, 2002, pp. 187-191.

[8] M.-O. Damen, H. El-Gamal, G. Caire, "On maximum likelihood detection and the search for the closest lattice point", IEEE Transactions on Information Theory, Vol. 49, No. 10, 2003, pp. 2389-2402.

[9] G. Romano, D. Ciuonzo, P. SalvoRossi, F. Palmieri, "Low-complexity dominance-based sphere decoder for MIMO systems", Signal processing, Vol. 93, No. 9, 2013, pp. 2500-2509.

[10] B. Trotobas, A. Nafkha, Y. Louët, "A review to massive MIMO detection algorithms: Theory and implementation", Advanced Radio Frequency Antennas for Modern Communication and Medical Systems, IntechOpen, London, UK, 2020.

[11] E. Larsson, "Mimo detection methods: How they work", IEEE Signal Processing Magazine, Vol. 26, No. 3, 2009, pp. 91-95.

[12] E. W. Dijkstra, "A note on two problems in connexion with graphs", Numerische mathematik, Vol. 1, No. 1, 1959, pp. 269-271.

[13] T. Datta, N. Srinidhi, A. Chockalingam, B. S. Rajan, "Random-restart reactive tabu search algorithm for detection in large-MIMO systems", IEEE Communications Letters, Vol.14, No. 12, 2010, pp.1107-1109.

[14] K. Su, "Efficient maximum likelihood detection for communication over multiple input multiple output channels", Department of Engineering, University of Cambridge, 2005.

[15] T. Fukatani, R. Matsumoto, T. Uyematsu, "Two methods for decreasing the computational complexity of the mimo $\mathrm{ml}$ decoder", IEICE Transac- 
tions on Fundamentals of Electronics, Communications and Computer Sciences, Vol. 87, No. 10, 2004, pp. 2571-2576.

[16] A. Okawado, R. Matsumoto, T. Uyematsu, "Near ml detection using dijkstra's algorithm with bounded list size over mimo channels", Proceedings of the IEEE International Symposium on Information Theory, Toronto, ON, Canada, 6-11 July 2008, pp. 2022-2025.

[17] R. Chang, W.-H. Chung, "Best-first tree search with probabilistic node ordering for mimo detection: Generalization and performance-complexity tradeoff", Wireless Communications, IEEE Transactions on, Vol. 11, No. 2, 2012, pp. 780-789.
[18] T.-H. Kim, I.-C. Park, "High-throughput and areaefficient mimo symbol detection based on modified dijkstra's search", IEEE Transactions on Circuits and Systems I: Regular Papers, Vol. 57, No. 7, 2010, pp. 1756-1766.

[19] W. Hou, T. Fujino, T. Kojima, "Adaptive candidate selection scheme in qrm-mld algorithm for mimo detection", Proceedings of the IEEE 8th International Conference on Wireless and Mobile Computing, Networking and Communications, Barcelona, Spain, 8-10 October 2012, pp. 502506. 